\title{
An Interrogation System Based on Two Semiconductor Optical Amplifiers for Weak Fiber Bragg Gratings
}

\author{
Zhihui $\mathrm{LUO}^{1,2}$, Hongqiao WEN ${ }^{1 *}$, and Huiyong GUO ${ }^{1}$ \\ ${ }^{1}$ National Engineering Laboratory of Fiber Optic Sensing Technology, Wuhan University of Technology, Wuhan, \\ 430070, China \\ ${ }^{2}$ China Three Gorges University, Yichang, 443002, China \\ *Corresponding author: Hongqiao WEN_Ｅ-mail: whq@whut.edu.cn
}

\begin{abstract}
An interrogation system based on two semiconductor optical amplifiers for weak fiber Bragg gratings (FBGs) was proposed. The first semiconductor optical amplifier (SOA) was used to modulate the light, and the second SOA separated the reflected signal from the different FBGs through delayed switching. The proposed system has lower insertion loss and higher spatial resolution, and can interrogate the time- and wavelength-division multiplexed FBG array. Up to 50 FBGs, with a reflectivity of $0.2 \%$ and a spatial resolution of $5 \mathrm{~m}$ along the optical fiber, were distinguished to demonstrate the interrogation system.
\end{abstract}

Keywords: Fiber Bragg grating, sensors, time-division multiplexing, semiconductor optical amplifiers, interrogation

Citation: Zhihui LUO, Hongqiao WEN, and Huiyong GUO, "An Interrogation System Based on Two Semiconductor Optical Amplifiers for Weak Fiber Bragg Gratings,” Photonic Sensors, 2014, 4(2): 168-172.

\section{Introduction}

Fiber Bragg gratings (FBGs) have attracted considerable interest for the quasi-distributed sensing network due to its high sensitivity, small size, wide dynamic range, and good reliability. The multiplexing capability, which is mainly determined by the interrogation method and the reflectivity of the FBG, is the performance parameter representing the monitor range and ability of the sensing network [1-3]. Wavelength-division multiplexing (WDM) becomes a popular technique due to its excellent wavelength demodulation characteristic. However, the WDM scheme is limited by the available bandwidth of broadband light sources, usually less than $120 \mathrm{~nm}$, and only accommodates tens of FBGs.
Time-division multiplexing (TDM) utilizes different time delays between the reflected pulses to distinguish the sensors with an identical wavelength and relieves the spectral bandwidth issue. Therefore, this method has the considerable potential for increasing the number of the multiplexing sensor. Several types of TDM schemes with a resonant cavity based on a semiconductor optical amplifier (SOA) were proposed [3-5]. Notably, these methods improve the signal power with a single SOA, and the interrogated FBGs with a reflectivity of more than $1 \%$ are required. However, for this normal FBG array, the multiplexing capacity is seriously limited by intrinsic crosstalk [6]. A newly developed optical time-domain reflectometry-FBG network with a weak FBG array has been reported [7, 8]. Its

Received: 14 December 2013 / Revised version: 30 December 2013

(C) The Author(s) 2014. This article is published with open access at Springerlink.com

DOI: $10.1007 / \mathrm{s} 13320-014-0168-1$

Article type: Regular 
interrogated system selects the tunable laser (T-LD) as the source and distinguishes FBGs by a high-speed data acquisition. Owing to the slow scanning speed of the T-LD, the real-time performance is poor.

The weak FBGs are very attractive because of its low crosstalk and the strong multiplexing capability. Recently, our team has successfully developed a FBG fabrication system which can perform on-line writing of large-scale FBG arrays during drawing fiber. This on-line writing FBG array has no fusion loss and high mechanical stability. Based on this weak FBG array, an interrogation system with two SOAs was proposed. The high power superluminescent light emitting diode (SLED) was selected as the light source, and one SOA was used to modulate the light into the pulse train, and the other was configured to separate the reflected pulses from different FBGs via delayed switching. The characteristic of SOAs was investigated, and the crosstalk of gratings was discussed. The proposed system has a low insertion loss and an excellent spatial resolution, and can interrogate the TDM/WDM FBG array.

\section{Operation principle}

The interrogation system for the weak FBG array is illustrated in Fig. 1. The continuous

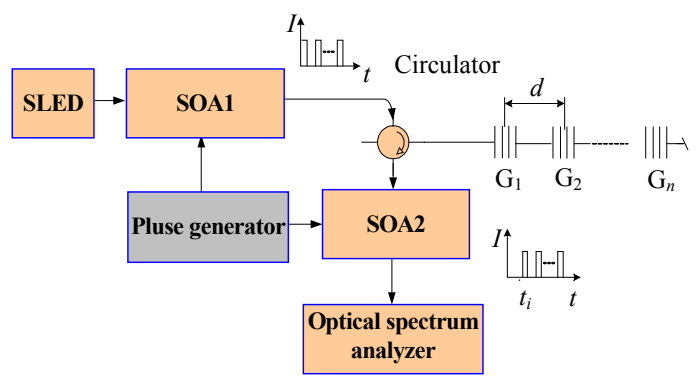

Fig. 1 Interrogation system for the weak FBG array.

wavelength (CW) light from an SLED(INPHENIX IPSDD1302) with a range of $1280 \mathrm{~nm}$ to $1340 \mathrm{~nm}$, is modulated and amplified into tens of nanosecond pulse train by the first SOA (SOA1, INPHENIX IPSAD1302). The pulses pass through the circulator and are launched into the serial TDM FBG array
$\left(G_{1}, \cdots, G_{n}\right)$, where $n$ is the number of FBGs in the array. Each FBG reflects part of the incident pulses back to the second SOA (SOA2, INPHENIX IPSAD1302) at a different time. When the SOA2 is "on", the pulses arriving at SOA2 are amplified, whereas other pulses are absorbed. The pulses that pass through SOA2 are detected by the optical spectrum analyzer (YOKOGAWA AQ6370) to obtain their wavelength shifts and intensities directly. Both SOAs are driven by two trains of homogeneous pulses from a two-channel generator programmed to adjust the delay time between the pulse trains. The time delay between pulse trains corresponds to the position of the fiber. The different FBGs in the array can be addressed separately by changing the time delay. To eliminate the signal overlapping, the delay time between two adjacent sensors is selected to be more than the pulse width. The relation between the time delay $\left(\tau_{i}, 1 \leqslant i \leqslant n\right)$ and the distance of FBGi from the $\operatorname{SOA}\left(L_{i}\right)$ is

$$
\begin{gathered}
\tau_{i}=\frac{2 n_{e} L_{i}}{c} \\
\left(\tau_{r}+\tau_{f}\right) \leq w \leq \frac{2 n_{e} d}{c}
\end{gathered}
$$

where $n_{e}$ is the effective refractive index of the fiber, $c$ is the speed of light in vacuum, $w$ is the pulse width, $\tau_{r}$ is the rise-time, and $\tau_{f}$ is the fall-time of the modulated pulse, which are associated with the form of the driving electrical pulse and the response time of the SOA. During the initialization of the system, the signal generator scans the delay time with a onenanosecond step, which corresponds to the spatial resolution of 0.1 meter along the fiber. The OSA detects the reflected signal and judges whether they are from FBGs by the preset intensity threshold and records the time delay that matches each FBG of the array. When the sensor network runs, the signal generator reads the recorded delays one by one and then adjusts the time delay between pulse trains. All FBGs are addressed in turns. Calculating the variation of the Bragg wavelength to the initial value, the measurement monitored by the FBG could be 
demodulated. Meanwhile, the spatial position of the current FBG could be obtained as (1). Here, the power of the SLED should be noted to be less than $10 \mathrm{dBm}$, so that the SOA1 works in an unsaturated state, at the same time that the power spectrum density (PSD) of the sensing wavelength should be more than $-28 \mathrm{dBm}$ to watch the good reflected signal from a weak FBG.

\section{Experimental results and discussion}

\subsection{Characteristic analysis of the SOA}

As we know that the SOA has an excellent characteristic of the high speed switch, usually its rise-time and fall-time are less than $1 \mathrm{~ns}$, respectively. Driven by the electric pulse, the SOA could modulate the $\mathrm{CW}$ light into ten of ns light pulse. The signal generator (RIGOL DG3072) is selected to produce two pulse trains with the same period and pulse width, which are homogeneous. At the $5-\mathrm{V}$ output peak voltage, the rise-time and fall-time $\left(\tau_{e r}=\right.$ $\left.\tau_{e f}\right)$ of the electric pulse are about $4 \mathrm{~ns}$. The voltage signal of the pulses is transformed the current by the constant current circuit to drive the SOA. The circuit has a response time of $1 \mathrm{~ns}$ for the rise edge $\left(\tau_{r r}\right)$ and $1.1 \mathrm{~ns}$ for the fall edge $\left(\tau_{r f}\right)$. Therefore, we can deduce the effective width of the optical pulse:

$$
w_{e}=w-\frac{1}{2}\left(\tau_{e r}+\tau_{e f}+\tau_{r r}+\tau_{r f}\right) .
$$

Given that the electrical pulse width is $20 \mathrm{~ns}$, the optical pulse modulated by the SOA are shown in Fig. 2. The bottom width of the optical pulse is $20 \mathrm{~ns}$, and the top width at the level of $3.3 \mathrm{~V}$ is about $6 \mathrm{~ns}$. About $13 \mathrm{~ns}$ of the full width at half maximum (FWHM) of the optical pulse is detected, which agrees with the calculated effective pulse width. In order to further improve the spatial resolution, compressing the pulse width is feasible. When the radio-frequency signal source is selected to generate the pulse narrower than $10 \mathrm{~ns}$, with rise-time and fall-time of less than $0.5 \mathrm{~ns}$, the resolution of $1 \mathrm{~m}$ should be achieved. Unfortunately, the cost of this system will increase sharply for this configuration. For the different input signal intensities, the gains of the SOA vary. When the input power of the SOA is less than $-10 \mathrm{dBm}$, the gain is $20 \mathrm{~dB}$ and then deceases sharply with an increase in the power of the input signal. Given that the $\mathrm{mW}$ level of the broadband light is selected as the light source, the SOA1 usually works under the saturated mode, and the gain is less than $5 \mathrm{~dB}$. Meanwhile, the output power is stable when the signal power from the light source varies slightly. Since the power of the reflected signal from the weak signal FBG is less than $-10 \mathrm{dBm}$, the SOA2 has a stable gain of $20 \mathrm{~dB}$. Therefore, the total gain of the signal from two SOAs is more than $25 \mathrm{~dB}$. In contrast to the electro-optic modulator (EOM) with the insertion loss of typical $5 \mathrm{~dB}$, this pioneering scheme has a lower insertion loss and an excellent cost.

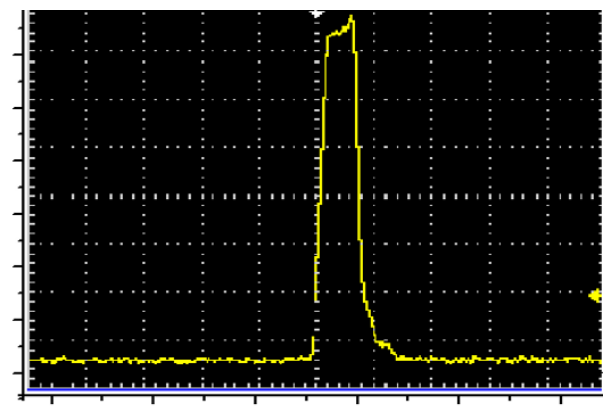

Fig. 2 Shape of the modulated light.

\subsection{Crosstalk of gratings}

For the TDM FBG array, the maximum number of interrogated FBGs is limited by two types of crosstalk, namely multiple-reflection crosstalk and spectral shadowing [7]. The multiple-reflection crosstalk refers to the spectral distortion induced by the false signal, which undergoes multiple reflections between the upstream FBGs and arrives at the detector at the same time with the real signal of downstream FBGs. Related studies show that the multiple-reflection crosstalk can be ignored when the reflectivity of the FBG is less than 1\% [7]. Spectral shadowing describes the spectral distortion 
of the downstream FBGs induced by the insertion loss of the upstream FBGs. For the weak FBG array, the returned power from the $i$ th $(i=1,2, \ldots, n) \mathrm{FBG}$ at the wavelength $\lambda$ can be approximated as $[8]$

$$
I_{r i}(\lambda)=I_{0}(\lambda) R(\lambda)[1-R(\lambda)]^{2(i-1)}
$$

where $I_{0}(\lambda)$ is the source spectrum, and $R(\lambda)$ is the reflection spectrum of the FBG. The higher the reflectivity of the FBG is, the more serious the spectral shadowing is, and then the much less the maximum number of multiplexed FBGs is. The relationship between the returning power and the reflectivity of the FBG is indicated in Fig. 3. Given that the sensor array includes 125 FBGs with the reflectivity of $0.2 \%(-27 \mathrm{~dB})$, the reflected power from the 125-th FBG will decline $2.17 \mathrm{~dB}$ as (4). The reflection spectra of the 1-th FBG (in the forward direction) from the two ends of the FBG array are shown in Fig. 4. The measured result

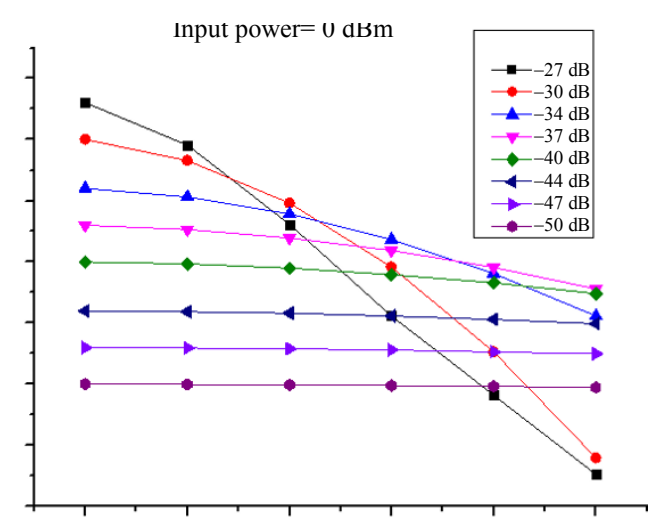

Fig. 3 Returning power of the serial FBG with different reflectivities.

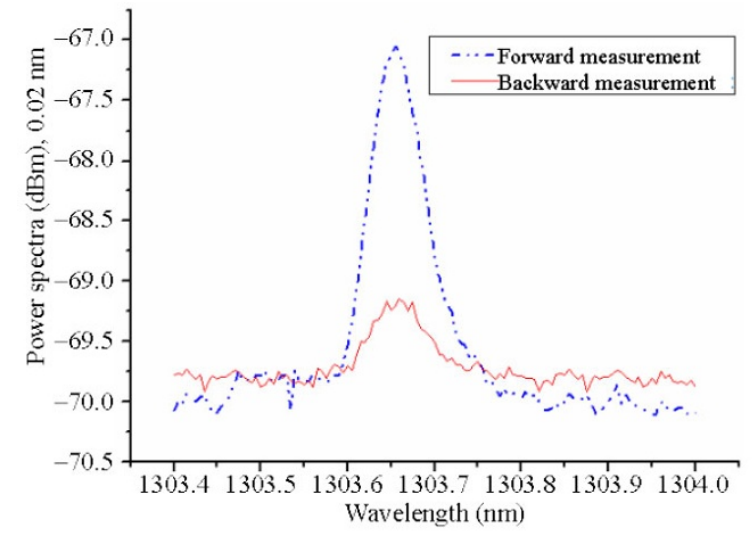

Fig. 4 Reflection spectra of the 1-th FBG from two ends of the FBG array. agrees with our theoretical calculation. Moreover, the reflection spectra of the 125-th (in the backward direction) becomes flattening for the shadowing effect, and the interrogated system is hard to search its peak wavelength.

\subsection{Interrogation experiment}

In order to further demonstrate the interrogation performance, $50 \mathrm{FBGs}$, with a reflectivity of $0.2 \%$ and a spatial resolution of $5 \mathrm{~m}$ along the optical fiber, were fabricated as a sensor array. Launching the pulse train with a pulse width of $20 \mathrm{~ns}$ and the frequency of $20 \mathrm{kHz}$, all FBGs were interrogated by changing the delay time. For easy to be observe, only the reflection spectra of 1-th, 2-th, 3-th, 4-th, 5 -th, 49-th, and 50-th are shown in Fig. 5. Corresponding to these FBGs, the delay time was $118 \mathrm{~ns}, 167 \mathrm{~ns}, 217 \mathrm{~ns}, 267 \mathrm{~ns}, 317 \mathrm{~ns}, 2570 \mathrm{~ns}$, and $2620 \mathrm{~ns}$, and the peak wavelengths were $1303.655 \mathrm{~nm}$, $1303.65 \mathrm{~nm}, 1303.640 \mathrm{~nm}, 1303.635 \mathrm{~nm}, 1303.630 \mathrm{~nm}$, $1303.67 \mathrm{~nm}$, and $1303.66 \mathrm{~nm}$, respectively. Here, the time delay of $118 \mathrm{~ns}$ for the 1-th FBG included the delay time which the light traveled in the optic pigtail. The difference of the delay time between two adjacent FBGs should be $50 \mathrm{~ns}$, and the error of less than $1 \mathrm{~ns}$ was mainly induced by the non-ideal shape of the modulated pulse. Due to the shadowing effect, the reflected signals from the 49-th FBG and the 50-th FBG were obviously weaker than the others. When the delay time of $167 \mathrm{~ns}$ was kept, the system monitored the 2nd FBG. A thermostat water

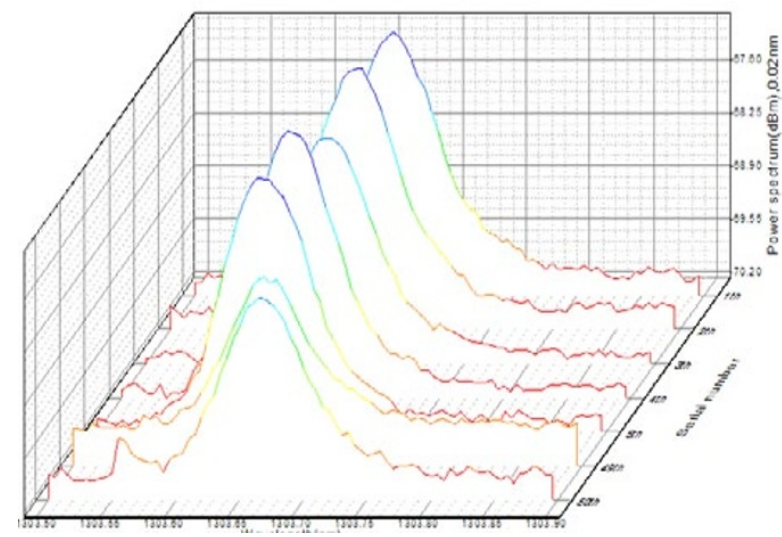

Fig. 5 Reflected signal from 14 FBGs. 
bath was used to adjust the temperatures. The measured peak wavelength as a function of the temperature is shown in Fig. 6, which indicates a sensitivity of $8.4 \mathrm{pm} /{ }^{\circ} \mathrm{C}$. Because the wavelength precision of $\mathrm{AQ6370B}$ is $1 \mathrm{pm}$, the temperature measuring precision of the 2-th FBG was $0.11{ }^{\circ} \mathrm{C}$.

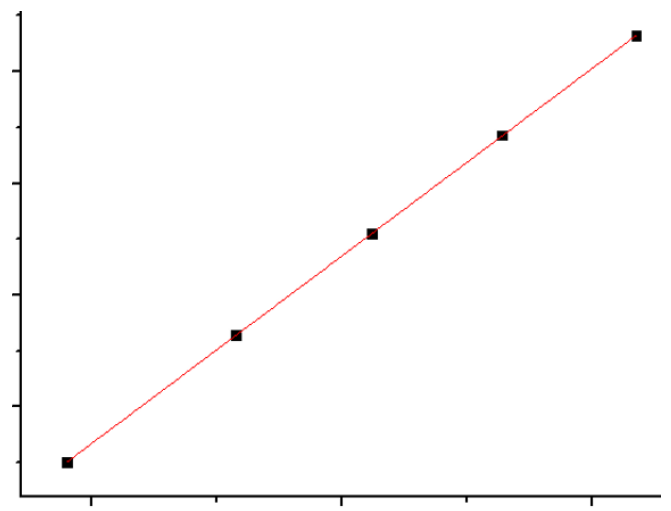

Fig. 6 Temperature sensing character of the 2-th FBG.

\section{Conclusions}

We have demonstrated an interrogation system based on two SOAs for the weak FBG array. The result shows that the interrogation system can distinguish 50FBGs with a spatial resolution of $5 \mathrm{~m}$ along the optical fiber. Owing to the broadband SLED configuration, the multiplexing capacity is increased by the traditional WDM technique. This interrogation scheme has a great potential for the large-scale FBG sensing network.

\section{Acknowledgment}

This work was supported in part by the National Science Foundation of China, NSFC (Grant No. 61205070), and the Major Program of the National Natural Science Foundation of China, NSFC (Grant No. 61290311).
Open Access This article is distributed under the terms of the Creative Commons Attribution License which permits any use, distribution, and reproduction in any medium, provided the original author(s) and source are credited.

\section{References}

[1] M. Zhang, Q. Sun, Z. Wang, X. Li, H. Liu, and D. Liu, "A large capacity sensing network with identical weak fiber Bragg gratings multiplexing," Optics Communications, 2012, 285(13-14), 3082-3087.

[2] D. J. F. Cooper, T. Coroy, and P. W. E. Smith, "Time-division-multiplexing of large serial fiber -optic Bragg grating sensor arrays," Applied Optics, 2001, 40(11), 2643-2554.

[3] J. Ou and Z. Zhou, "Optic fiber Bragg-grating-based sensing technologies and their applications in structural health monitoring," in Proc. SPIE, vol. 6595, 726612-1-726612-8, 2007.

[4] W. H. Chung, H. Y. Tam, P. K. A. Wai, and A. Khandelwal, "Time- and wavelength-division multiplexing of FBG sensors using a semiconductor optical amplifier in ring cavity configuration," IEEE Photonics Technology Letters, 2005, 17(12): 2709-2711.

[5] G. D. Lloyd, L. Bennion, L. A. Everall, and K. Sugden, "Novel resonant cavity TDM demodulation scheme for FBG sensing," in Conference on Lasers and Electro-Optics, San Francisco, California, May 16-21, 2004.

[6] Y. Dai, Y. Liu, J. Leng, G. Deng, and A. Asundi, “A novel time-division multiplexing fiber Bragg grating sensor interrogator for structural health monitoring," Optics and Lasers in Engineering, 2009, 47(10): 1028-1033, 2009.

[7] Y. Wang, J. Gong, D. Wang, B. Dong, W. Bi, and A. Wang, "A quasi-distributed sensing network with time-division-multiplexed fiber Bragg gratings," IEEE Photonics Technology Letter, 2011, 23(2): 70-72.

[8] Y. M. Wang, J. M. Gong, D.Y. Wang, T. J. Shilig, and A. Wang, "A large serial time-division multiplexed fiber Bragg grating sensor network," Lightwave Technology, 2012, 30(17): 2751-2756. 